\title{
Estudio observacional y descriptivo de prevalencia de onicomicosis en una población que acude a una consulta de dermatología del Área Sanitaria de Ferrol
}

\section{Observational and descriptive study of prevalence of onychomycosis in a population attending a dermatology office in the Health Area of Ferrol}

\author{
Cristina Martínez Vilar ${ }^{1}$, Abián Mosquera Fernández ${ }^{2}$, Benigno Monteagudo Sánchez ${ }^{3}$ \\ ${ }^{1}$ Grado en Podología. Universidade da Coruña \\ crismartinezvi@hotmail.com \\ ${ }^{2}$ Profesor colaborador doctor. Departamento de Ciencias de la Salud. Facultad de Enfermería y Podología. Universi- \\ dade da Coruña, Campus de Ferrol \\ abian@udc.es \\ ${ }^{3}$ Licenciado en Medicina y Cirugía. Servicio de Dermatología. Complejo Hospitalario Universitario de Ferrol. Área \\ Sanitaria de Ferrol-Servicio Gallego de Salud \\ benigno.monteagudo.sanchez@sergas.es
}

Correspondencia:

Cristina Martínez Vilar

C/ Manuel Fernández Avello no 8, $4^{\circ} \mathrm{A}$

E-33011 Oviedo

Correo electrónico: crismartinezvi@hotmail.com

Fecha de recepción: 9 septiembre de 2014

Fecha de aceptación: 23 de octubre de 2014

Los autores declaran no tener ningún tipo de interés económico o comercial.

\section{RESUMEN}

Objetivos: Detarminar la prevalencia de onicomicosis en una muestra de personas que acuden a una consulta de dermatología y estudiar cómo influyen diversos factores personales en la frecuencia de aparición de esta enfermedad. Metodología: Se seleccionó una muestra de 100 sujetos (42 hombres y 58 mujeres) atendidos de forma consecutiva durante el periodo de estudio. Se realizó un diagnóstico clínico de la patología basado en los signos clínicos observados durante la exploración de las extremidades inferiores.

Resultados: El 18\% de los participantes presentó onicomicosis, de los cuales el 61,1\% fueron mujeres y el 38,9\% hombres. Las enfermedades asociadas más prevalentes que presentaban las personas con onicomicosis fueron por este orden las de tipo dermatológico $(16,7 \%$ ) y las de tipo cardiovascular y endocrino (ambas con un $11,1 \%$ ).

La tinea pedis estuvo presente en el $33,3 \%$ de aquellos que presentaban onicomicosis no existiendo diferencias en los porcentajes entre tinea pedis plantar e interdigital. El tipo de onicomicosis más frecuente fue la subungueal distal (en el $100 \%$ de los casos) y el primer dedo del pie resultó ser el más afectado.

Conclusiones: Se ha obtenido una presvalencia de onicomicosis consistente con lo publicado en la literatura actual. Nuestros resultados confirman la relación entre las enfermedades concomitantes encontradas y la presencia de tinea pedis con la presencia de la onicomicosis.

Palabras clave: epidemiología; onicomicosis; tinea unguium.

\section{ABSTRACT}

Objectives: To determine the prevalence of onychomycosis in a sample of subjects attending a dermatology office and to study how different personal factors influence the frequency of occurrence of this disease.

Methods: This study comprised a sample of 100 subjects ( 42 men and 58 women) attended consecutively during the course of the study. A clinical diagnosis of the disease was performed based on clinical signs observed during physical examination of the lower extremities.

Results: $18 \%$ of the participants had onychomycosis, of whom $61.1 \%$ were women and $38.9 \%$ were men. The most prevalent associated diseases in subjects with onychomycosis were in this order dermatological type diseases $(16.7 \%)$ 
and cardiovascular and endocrine type diseases (both with 11.1\%). Tinea pedis was present in $33.3 \%$ of those with onychomycosis with no difference in percentage between plantar and interdigital tinea pedis. The most common type of onychomycosis was the distal subungueal type (in 100\% of the cases) and the great toe was the most affected one. Conclusions: A prevalence of onychomycosis consistent with the published current literature has been obtained. Our results confirm the relationship between comorbidities found and the presence of tinea pedis with the presence of onychomycosis.

Keywords: epidemiology; onychomycosis; tinea unguium.

Sumario: 1. Introducción. 2. Objetivo general. 3. Objetivos específicos e hipótesis asociadas. 4. Material y Métdoos. 5. Resultados. 6. Discusión y conclusiones. Bibliografía.

Referencia normalizada: Lasanta Lacalzada, L., Reyes Martín, R. Estudio observacional y descriptivo de prevalencia de onicomicosis en una población que acude a una consulta de dermatología del Área Sanitaria de Ferrol. Rev. Int. Cienc. Podol. 2015; 9(2): 89-98. 


\section{INTRODUCCIÓN}

La onicomicosis (ONM) es la infección de las uñas ocasionada por hongos levaduriformes o filamentosos y constituye una de las patologías ungueales más frecuentes, ya que suponen entre el $15 \%$ y el $40 \%$ de las onicopatías ${ }^{1}$. Esta afección es más frecuente en las uñas de los pies y se estima que afecta entre el $2 \%$ y el $9 \%$ de la población mundial ${ }^{2}$. Su frecuencia está relacionada con factores predisponentes, distintas patologías de base, clase social, ocupación, edad y clima ${ }^{3}$; y su prevalencia aumenta con la edad, el sexo masculino, la presencia de tinea pedis, estados de inmunodeficiencia, alteraciones circulatorias y la Diabetes Mellitus (DM) $)^{4,5}$. Las guías clínicas vigentes recomiendan confirmar el diagnóstico antes de iniciar un tratamiento sistémico mediante examen directo con hidróxido de potasio $(\mathrm{KOH})$ de fragmentos ungueales, cultivo o biopsia de la uña con estudio anatomopatológico. Sin embargo, estas pruebas no tienen toda la eficacia que pudiera desearse ya que presentan un valor predictivo positivo de alrededor del $75 \%$ y un valor predictivo negativo que oscila entre el $67 \%$ y el $90 \%$. Por este motivo, en ocasiones se puede confiar más en los signos clínicos observados que en los resultados obtenidos por dichas pruebas ${ }^{6}$.

En la actualidad existen varios estudios que intentan reunir los signos clínicos ungueales específicos para establecer un diagnóstico clínico de ONM más preciso y de esta manera poder descartar otras patologías ungueales ${ }^{7,8}$.

\section{OBJETIVO GENERAL}

Conocer la prevalencia de ONM en una muestra de personas atendidas en una consulta de dermatología sin diagnóstico previo o sospecha diagnóstica y analizar cómo influyen determinados factores personales en la frecuencia de aparición de esta afección.

\section{OBJETIVOS ESPECÍFICOS E HIPÓ- TESIS ASOCIADAS}

Objetivo específico 1: Analizar la prevalencia de ONM en función de la edad en la muestra estudiada.
Hipótesis asociada: Los mayores de 65 años presentan una prevalencia significativamente superior a la que presentan las personas con edad inferior a ésta.

Objetivo específico 2: Analizar la prevalencia de ONM en función del sexo.

Hipótesis asociada: No se esperan diferencias estadísticamente significativas entre ambos sexos.

Objetivo específico 3: Conocer si existe relación entre presentar ONM y enfermedades de tipo dermatológico, hematológico, digestivas, vasculares, genéticas, endocrinas, de medicina interna e inmunológicas.

Hipótesis asociada: Se espera que exista una relación estadísticamente significativa entre presentar ONM y enfermedades de tipo dermatológico, vasculares, endocrinas e inmunológicas. Se espera que no exista una relación estadísticamente significativa entre presentar ONM y enfermedades de tipo hematológico, digestivas, genéticas y de medicina interna.

Objetivo específico 4: Identificar la relación que existe entre presentar ONM y padecer $t i-$ nea pedis.

Hipótesis asociada: Se espera que exista una relación estadísticamente significativa.

Objetivo específico 5: Conocer la prevalencia de ONM según su localización y tipo.

Hipótesis asociada: Se espera que la mayor prevalencia de ONM se encuentre localizada en el primer dedo del pie. Se espera que el tipo de ONM más prevalente sea la subungueal distal. Se espera que existan diferencias estadísticamente significativas entre pie izquierdo y pie derecho.

\section{MATERIAL Y MÉTODO}

\section{1. Ámbito y población de estudio}

Personas que acuden a una consulta de dermatología del Hospital Naval de Ferrol con afecciones dérmicas que podrían estar asociadas a otro tipo de patologías (endocrinas, hematológicas, digestivas, vasculares, genéticas, medicina interna e inmunológicas).

\subsection{Tipo de estudio}

Estudio transversal, observacional y descriptivo. 


\subsection{Muestreo}

Se seleccionó una muestra de 100 sujetos atendidos de forma consecutiva durante el periodo de estudio.

\subsection{Criterios de inclusión y exclusión}

Fueron incluidas personas atendidas en una consulta de dermatología del Hospital Naval de Ferrol con una edad igual o superior a 2 años. Se excluyeron aquellas ya diagnosticadas o remitidas con sospecha de ONM o tinea pedis.

\subsection{Variables estudiadas}

Se recogieron las variables edad, sexo, presencia de ONM (incluyendo tipo y localización), presencia de tinea pedis y las enfermedades asociadas que se enumeran a continuación:

1) Dermatológicas: psoriasis y pénfigo vulgar.

2) Hematológicas: policitemia vera y linfoma.

3) Digestivas: carcinoma gástrico.

4) Vasculares: insuficiencia venosa.

5) Genéticas: Síndrome de Down.

6) Endocrinas: DM y síndrome de Cushing.

7) Medicina interna: lupus eritematoso sistémico.

8) Inmunológicas: enfermedades inmunodepresivas.

Las variables edad y sexo fueron operativizadas en las siguientes categorías:

- Edad: 1) 0-31 años; 2) 32-65 años y 3) 66-99 años.

- Sexo: 1) Hombre y 2) Mujer.

\subsection{Bases de datos consultadas}

Se consultaron las bases de datos de Pubmed, Medline, Dialnet y biblioteca Cochrane de las cuales se recogieron los artículos publicados desde el año 2000 hasta la actualidad.

\subsection{Consideraciones éticas}

El estudio se desarrolló con el permiso del servicio de dermatología del Hospital Naval de Ferrol y siguió en todo momento las normas éticas recogidas en los reglamentos internacionales para la evaluación ética de los estudios epidemiológicos y de la investigación biomédica en humanos. La información referente a la identificación de los participantes fue considerada confidencial a todos los efectos de forma que su identidad no pudo ser desvelada ni divulga- da siguiendo lo establecido en la Ley Orgánica 15/1999 de 13 de diciembre de "protección de datos de carácter personal". Se informó a los participantes de la finalidad del estudio y se obtuvo su consentimiento informado respetando su derecho a la intimidad.

\subsection{Análisis estadístico}

Las variables edad, sexo, presencia de ONM (incluyendo tipo y localización), presencia de tinea pedis y presencia de enfermedades asociadas (dermatológicas, hematológicas, digestivas, vasculares, genéticas, endocrinas, de medicina interna e inmunológicas) se analizaron con el paquete estadístico SPSS 22.0 a través de los siguientes procedimientos de análisis:

- Frecuencias y porcentajes: para calcular la prevalencia de ONM en función de la edad, del sexo y de la localización y tipo de ONM en la muestra estudiada.

- Índice de asociación Chi-cuadrado: para analizar si existe relación entre "presentar ONM y alguna de las enfermedades asociadas mencionadas anteriormente" o padecer tinea pedis.

\section{RESULTADOS}

\subsection{Descripción de la muestra en función de las variables de estudio}

Tras el análisis observamos que del total de sujetos estudiados, un $18 \%$ presentó ONM y un $6 \%$ tinea pedis. La edad media de los participantes fue de 47,3 años con una mayoría (48\%) presentando una edad comprendida entre los 32 y los 65 años. Con respecto al sexo, un $42 \%$ de participantes fueron hombres y un 58\% mujeres. Por su parte y en referencia a las enfermedades asociadas observamos que la mayoría de participantes no presentó enfermedad asociada alguna $(76 \%)$ mientras que de aquellos que sí presentaron vemos que las enfermedades más frecuentes fueron las de tipo endocrino $(8 \%)$ seguidas por las de tipo dermatológico $(7 \%)$, vasculares $(4 \%)$, de medicina interna $(2 \%)$ y por último con igual frecuencia genéticas y hematológicas $(1 \%)$.

En cuanto a la distribución porcentual del tipo y localización de ONM, la totalidad de afectados por ONM presentó una ONM de tipo subungueal distal siendo el primer dedo el más afectado. 


\subsection{Prevalencia de ONM en función de la edad}

El valor obtenido tanto de Chi-cuadrado $(2,281)$ como del coeficiente de contingencia $(0,149)$ no han resultado estadísticamente significativos $(\mathrm{p}=0,32)$ (véase Tabla 1) lo que indica que en la muestra estudiada no hay asociación entre la edad y presentar ONM. No obstante, el análisis porcentual muestra una mayor prevalencia de ONM en personas con edades comprendidas entre los 32 y los 65 años $(55,6 \%$ ), seguida del grupo de personas de entre 66 y 99 años $(33,3 \%)$ y por último del grupo de personas más jóvenes con solo un $11,1 \%$. Estos resultados permiten dar respuesta al objetivo específico 1 y rechazar su hipótesis asociada ("las personas mayores de 65 años presentan una prevalencia significativamente superior a la que presentan las personas con edad menor a ésta").

\subsection{Prevalencia de ONM en función del sexo} Los valores de significación de Chi-cuadrado $(0,087)$ y del coeficiente de contingencia $(0,768)$ (véase Tabla 2) permiten dar respuesta al objetivo específico 2 y aceptar su hipótesis asociada ("no se esperan diferencias estadísticamente significativas entre ambos sexos en la prevalencia de ONM"). No obstante, la distri- bución porcentual obtenida indica que del total de personas que presentan ONM se observa una mayor tendencia entre las mujeres $(61,1 \%)$ que entre los hombres $(38,9 \%)$.

\subsection{Prevalencia de ONM en función de la presencia de enfermedades asociadas (der- matológicas, hematológicas, digestivas, vas- culares, genéticas, endocrinas, de medicina interna e inmunológicas)}

Los valores de Chi-cuadrado y del coeficiente de contingencia han resultado estadísticamente significativos en ambos casos $(0,007)$ (véase Tabla 3). En nuestra muestra los sujetos que presentan ONM tienden también a mostrar en este orden enfermedades de tipo dermatológico $(16,7 \%)$, enfermedades endocrinas y vasculares $(11,1 \%$ en ambos casos). Este resultado permite dar respuesta al objetivo específico 3 y confirmar su hipótesis asociada ("se espera que exista una relación estadísticamente significativa entre presentar ONM y enfermedades de tipo dermatológico, vasculares, endocrinas e inmunológicas". "Se espera que no exista una relación estadísticamente significativa entre presentar ONM y enfermedades de tipo hematológico, digestivas, genéticas y de medicina interna").

Tabla 1. Prevalencia de ONM en función de la edad.

\begin{tabular}{c|cc}
\hline EDAD & \multicolumn{2}{|c}{ ONICOMICOSIS } \\
& NO & SI \\
\hline 0-31 años & $28 \%$ & $11,1 \%$ \\
32-65 años & $46,3 \%$ & $55,6 \%$ \\
66-99 años & $25,6 \%$ & $33,3 \%$ \\
\hline
\end{tabular}

\begin{tabular}{c|cc}
\hline \multicolumn{3}{c}{ Pruebas de chi-cuadrado } \\
\hline & Valor & Sig. asintótica \\
\hline Chi-cuadrado de Pearson & 2,281 &, 320 \\
\hline \multicolumn{3}{c}{ Medidas simétricas } \\
\hline & Valor & Aprox. Sig. \\
\hline Coeficiente de contingencia &, 149 &, 320 \\
\hline
\end{tabular}


Tabla 2. Prevalencia de ONM en función del sexo.

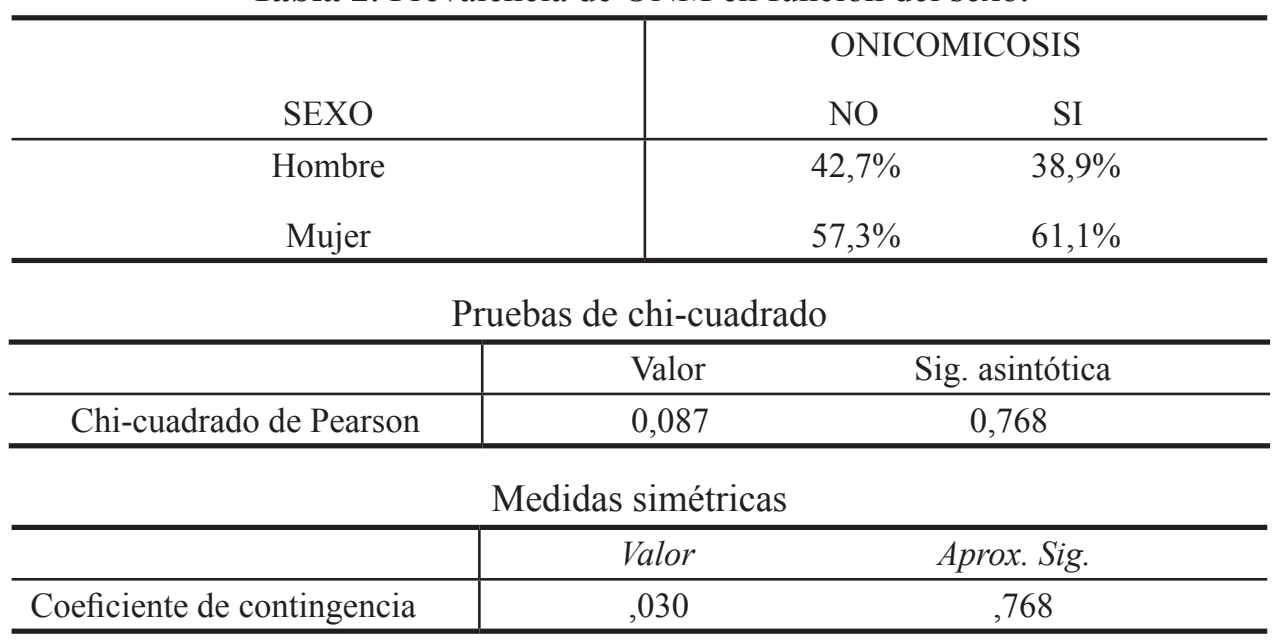

Tabla 3. Prevalencia de ONM en función de las enfermedades asociadas.

\begin{tabular}{c|cc}
\hline ENFERMEDADES & \multicolumn{2}{|c}{ ONICOMICOSIS } \\
ASOCIADAS & NO & SI \\
\hline Digestivas & $1,2 \%$ & $0,0 \%$ \\
Vasculares & $2,4 \%$ & $11,1 \%$ \\
Dermatológicas & $4,9 \%$ & $16,7 \%$ \\
Endocrinas & $7,3 \%$ & $11,1 \%$ \\
Genéticas & $0,0 \%$ & $5,6 \%$ \\
Hematológicas & $0,0 \%$ & $5,6 \%$ \\
Medicina interna & $1,2 \%$ & $5,6 \%$ \\
\hline
\end{tabular}

Pruebas de chi-cuadrado

\begin{tabular}{c|cc}
\hline & Valor & Sig. asintótica \\
\hline Chi-cuadrado de Pearson & 19,565 &, 007 \\
\hline
\end{tabular}

Medidas simétricas

\begin{tabular}{c|cc}
\hline & Valor & Aprox. Sig. \\
\hline Coeficiente de contingencia &, 405 &, 007 \\
\hline
\end{tabular}




\subsection{Prevalencia de ONM en función de la presencia o no de tinea pedis}

En este caso y al igual que en el apartado anterior los valores de Chi-cuadrado y del coeficiente de contingencia son estadísticamente significativos $(0,000)$ (véase Tabla 4) con lo que podemos responder al objetivo específico 4 y aceptar la hipótesis asociada ("se espera que exista una relación estadisticamente significativa entre presentar ONM y tinea pedis"). De aquellas personas que presentaron ONM un $11,1 \%$ tuvo además tinea pedis interdigital y en un mismo porcentaje tinea pedis plantar.

\subsection{Prevalencia de ONM en función de su tipo y localización}

También en este caso los valores de Chi-cuadrado y del coeficiente de contingencia son estadísticamente significativos $(0,000)$ (véase Tabla 5) lo que nos permite dar respuesta al objetivo específico 5 y aceptar su hipótesis asociada ("se espera que la mayor prevalencia de ONM se encuentre localizada en el primer dedo del pie. Se espera que el tipo de ONM más prevalente sea la subungueal distal. Se espera que existan diferencias estadisticamente significativas entre pie izquierdo y pie derecho"). De las personas estudiadas con ONM la totalidad muestra una afectación de tipo subungueal distal. Un 50\% de los casos se localizó en el primer dedo de uno de los pies y un $33,3 \%$ en el primer dedo pero de forma bilateral, resultando el pie izquierdo el más afectado $(61,2 \%)$.

\section{DISCUSIÓN Y CONCLUSIONES}

A nivel mundial la ONM afecta a entre un 5\% y un $20 \%$ de la población y se sabe que su frecuencia aumenta con la edad ${ }^{4,12,18,22}$. En algunos trabajos la muestra seleccionada la conforman personas con sospecha de $\mathrm{ONM}^{12,16}$ mientras que em otros se incluyen sujetos con problemas dérmicos o ungueales pudiendo ser el motivo de consulta padecer $\mathrm{ONM}^{2}$. Diferentes autores consideran además la ONM una patología de difícil diagnóstico y tratamiento que también afecta al individuo a nivel social y emocional con frecuentes recidivas que generan un gasto considerable para el sistema sanitario y constituyen algo más que un simple problema estético ${ }^{2,17}$.

En este estudio se realizó un diagnóstico clínico de la ONM a diferencia de otros estudios que realizan examen micológico ${ }^{3,4,11-13}$. Para ello se excluyeron todas aquellas personas cuyo motivo de consulta fuera la ONM por lo que ésta se diagnosticó de forma casual al igual que ha ocurrido en trabajos llevados a cabo en América del Sur y del Norte ${ }^{13}$, Asia y Europa. Se optó por este criterio diagnóstico debido a que existen estudios en los que las pruebas

Tabla 4. Prevalencia de ONM en función de la presencia o no de tinea pedis.

\begin{tabular}{|c|c|c|c|}
\hline \multirow[b]{2}{*}{ TINEA PEDIS } & \multicolumn{3}{|c|}{ ONICOMICOSIS } \\
\hline & & NO & SI \\
\hline $\begin{array}{r}\text { Interdigital } \\
\text { Plantar }\end{array}$ & & $\begin{array}{l}0,0 \% \\
0,0 \%\end{array}$ & $\begin{array}{l}11,1 \% \\
11,1 \%\end{array}$ \\
\hline \multicolumn{4}{|c|}{ Pruebas de chi-cuadrado } \\
\hline & Valor & & Sig. asintótica \\
\hline Chi-cuadrado de Pearson & 29,078 & & 000 \\
\hline \multicolumn{4}{|c|}{ Medidas simétricas } \\
\hline & Valor & & Aprox. Sig. \\
\hline Coeficiente de contingencia & ,475 & &, 000 \\
\hline
\end{tabular}


Tabla 5. Prevalencia de ONM en función de su tipo y localización.

\begin{tabular}{c|cc}
\hline & \multicolumn{2}{|c}{ ONICOMICOSIS } \\
TIPO Y LOCALIZACIÓN & NO & SI \\
\hline Subungueal distal & $0,0 \%$ & $100 \%$ \\
Primero a cuarto dedo pie izquierdo & & $5,6 \%$ \\
Cuarto dedo pie izquierdo & $0,0 \%$ & $5,6 \%$ \\
Primer dedo pie izquierdo & $0,0 \%$ & $44,4 \%$ \\
Segundo dedo pie izquierdo & $0,0 \%$ & $5,6 \%$ \\
Primer dedo pie derecho & $0,0 \%$ & \\
& & $5,6 \%$ \\
Primer dedo ambos pies & $0,0 \%$ & $33,3 \%$ \\
\hline
\end{tabular}

Pruebas de chi-cuadrado

\begin{tabular}{c|cc}
\hline & Valor & Sig. asintótica \\
\hline Chi-cuadrado de Pearson & 100,000 &, 000 \\
\hline & Medidas simétricas \\
\hline & Valor & Aprox. Sig. \\
\hline Coeficiente de contingencia &, 707 &, 000 \\
\hline
\end{tabular}

diagnósticas (examen directo con hidróxido de potasio, cultivo o biopsia) presentan un valor predictivo positivo de alrededor del $75 \%$ y un valor predictivo negativo que oscilaría entre el $67 \%$ y el $90 \% 6$.

Hemos hallado una prevalencia de ONM del 18\% similar a la obtenida en América del Sur $(18,8 \%)^{13}$, excepto Argentina y el Noroeste de Brasil cuya cifra es considerablemente inferior, al igual q ocurre en países como la India (45\%$61 \%)^{3,4,12}$. Sin embargo este dato es superior al hallado en lugares como Camerún $(8,8 \%)$, América del Norte $(8,9 \%)$ y Asia $(12,1 \%)^{13-}$ 15,18 .

En la muestra estudiada la mayor prevalencia de ONM se observó en el grupo de entre 32 y 65 años de edad $(55,6 \%)$ no encontrándose una relación estadísticamente significativa en- tre la edad y la presencia de dicha patología, datos semejantes a los publicados en Brasil y Camerún ${ }^{11,12,15}$. Por el contrario otros estudios hallan una mayor prevalencia de ONM entre los 10 y los 40 años (70\%) o entre mayores de 60 años $(46 \%)^{2,16,18-20}$.

Varios trabajos llevados a cabo en América del Norte, Islandia, Dinamarca y México muestran que esta patología es más común entre los hombres ${ }^{5,13,14,20,21}$ sin embargo nuestros resultados mostraron una mayor tendencia a presentarse en mujeres $(61,1 \%)$ al igual que ha ocurrido en otras investigaciones s, $^{2,11,12}$.

Se sabe que la prevalencia de ONM puede venir determinada por factores como la edad, el sexo, la ocupación, la clase social, la presencia de enfermedades como la $\mathrm{DM}^{4,5,11,14,18}$ etc. De hecho en nuestro trabajo hemos observa- 
do una relación entre presentar enfermedades asociadas y la presencia de ONM. Las enfermedades concomitantes más frecuentes fueron por este orden las afecciones dermatológicas, endocrinas y las de tipo cardiovascular. Algunos artículos describen una prevalencia de ONM en diabéticos de entre el $22 \%$ y el $52,8 \%$ $\mathrm{y}$ en pacientes con patología dérmica de entre el $12,7 \%$ y el $21,5 \%^{2,21,22}$. La coexistencia de ONM e infecciones fúngicas de la piel es actualmente un fenómeno frecuente siendo la más común la tinea pedis lo que seguramente se pueda atribuir a la propagación del hongo de la piel al aparato ungueal.

Hemos observado una prevalencia de tinea pedis entre los afectados por ONM del 33,3\% encontrándose la misma proporción de tinea pedis plantar e interdigital. Otros estudios muestran una prevalencia similar de esta patología ${ }^{16,23}$ y una mayor frecuencia en la planta del pie $(23,4 \%)$, frente a la forma interdigital $(10,9 \%)^{5}$.

En nuestro estudio el tipo de ONM más común fue la subungueal distal y lateral preva- lente en la totalidad de los casos como también ha ocurrido en otras ocasiones s, $^{2,5,16}$. La localización más frecuente fue el primer dedo de un pie $(9 \%)$ seguida del primer dedo en ambos pies $(6 \%)$ como también se ha visto en Brasil y otros lugares de España ${ }^{2,14}$. El 61,2\% presentó la afectación en el pie izquierdo, seguido de una afectación bilateral $(33,3 \%)$ semejante a lo obtenido en un estudio brasileño ${ }^{2}$.

En conclusión, la prevalencia de ONM obtenida es consistente con lo publicado en la literatura científica siendo el tipo subungueal distal y el primer dedo del pie la forma y localización más frecuentes. Además, en la muestra estudiada se ha demostrado que las enfermedades concomitantes guardan relación con la presencia de esta patología. A la vista de estos resultados creemos que sería recomendable ampliar el tamaño muestral lo que supondría una mayor representación de la población y ya que se ha demostrado que ciertas patologías podrían determinar la presencia de ONM sería aconsejable además tratar de evitar su aparición tomando las medidas preventivas necesarias.

\section{BIBLIOGRAFÍA}

1. Domingo Herranz H, Ribera Pibernat M. Onicopatías más frecuentes en Atención Primaria. Jano. 2005; 69(1568): 49-57.

2. Di Chiacchio N, Madeira CL, Suarez MV, Refkalefsky Loureiro W. An observational and descriptive study of the epidemiology of and therapeutic approach to onychomycosis in dermatology offices in Brazil. An Bras Dermatol. 2013; 88(1): 3-11.

3. Relloso S, Arechavala A, Guelfand L, Maldonado I, Walker L, Agorio I, Reyes S, Giusiano G, Rojas F, Flores V, Capece P, Posse G, Nicola F, Tutzer S, Bianchi M. Onicomicosis: estudio multicéntrico clínico, epidemiológico y micológico. Rev Iberoam Micol. 2012; 29:157-63.

4. Kaur R, Kashyap B, Bhalla P. Onychomycois - epidemiology, diagnosis and management. Indian J Med Microbiol. 2008; 26: 108-16.

5. Chanussot $\mathrm{C}$, Arenas R. Infección micótica plantar e interdigital en pacientes con onicomicosis. Rev Iberoam Micol. 2007; 24: 118-21.

6. Aranegui B, García-Doval I, Cruces M. Actitud de los dermatólogos ante lesiones sugestivas de onicomicosis en los pies. Actas Dermosifiliogr. 2009; 100: 342-3.

7. García-Doval I, Cabo F, Monteagudo B, Álvarez J, Ginarte M, Rodríguez-Álvarez MX, Abalde MT, Fernández ML, Allegue F, Pérez-Pérez L, Flórez A, Cabanillas M, Peón G, Zulaica A, del Pozo J. Clinical diagnosis of toenail onychomycosis is possible in some patients: cross-sectional diagnostic study and development of a diagnostic rule. Br J Dermatol. 2010; 163: 743-51.

8. Ginarte M, García-Doval I, Monteagudo B, Cabanillas M, Labandeira J, Flórez A, Cabo F, Álvarez J, Zulaica A, Allegue F, Pérez L, Abalde MT, Rosón E, de la Torre C, Rodríguez MX. Observer agreement in toenail disorders: implications for diagnosis and clinical research. Br J Dermatol. 2009; 160: 1315-7.

9. Hernández Ávila M, Garrido Latorre F, López Moreno S. Diseño de estudios epidemiológicos. Salud Pública Méx. 2000; 42(2): 144-54.

10. García Salinero J. Estudios descriptivos. Nure investigación [revista en internet] 2004 [acceso el 4 de mayo de 2014]. Disponible en: http://www.fuden.es/ficheros_administrador/f_metodologica/formacion $\% 207 . p d f$ 
11. Alves Martins E, Vieira Guerrer L, Cássia Cunha K, Costa Nunes Soares MM, Gottardo de Almeida MT. Onychomycosis: clinical, epidemiological and mycological study in the municipality of Sao José do Rio Preto. Rev Soc Bras Med Trop. 2007; 40(5): 1-4.

12. Brilhante RSN, Cordeiro RA, Medrano DJA, Rocha MFG, Monteiro AJ, Cavalcante CSP, et al. Onychomycosis in Ceará (Northeast Brazil): epidemiological and laboratory aspects. Mem Inst Oswaldo Cruz. 2005; 100(2): 1-7.

13. Sigurgeirsson B, Baran R. The prevalence of onychomycosis in the global population - a literature study. J Eur Acad Dermatol Venereol. 2013. DOI: 10.1111/jdv.12323.

14. Perea S, Ramos MJ, Garau M, Gonzalez A, Noriega AR, del Palacio A. Prevalence and risk factors of tinea unguium and tinea pedis in the general population in Spain. J Clin Microbiol. 2000; 38(9): 3226-30.

15. Nkondjo Minkoumou S, Fabrizi V, Papini M. Onychomycosis in Cameroon: a clinical and epidemiological study among dermatological patients. Int J Dermatol. 2012; 51(12): 1474-7.

16. Neupane S, Pokhrel DB, Pokhrel BM. Onychomycosis: a clínico-epidemiological study. Nepal Med Coll J. 2009; 11(2): 92-5.

17. Arrese JE, Valverde JC, Pierard GE. Un nuevo enfoque sobre la epidemiología de las onicomicosis. Rev Iberoam Micol. 2005; 22: 163-6.

18. Scher RK, Rich P, Pariser D, Elewski B. The epidemiology, etiology, and pathophysiology of onychomycosis. Semin Cutan Med Surg. 2013; 32(2 Suppl 1):S2-S4.

19. Bramono K, Budimulja U. Epidemiology of onychomycosis in Indonesia: data obtained from three individual studies. J Med Mycol. 2005; 46: 171-6.

20. Sigurgeirsson B, Steingrímsson O, Sveinsdóttir S. Prevalence of onychomycosis in Iceland: a population-based Study. Acta Derm Venereol. 2002; 82: 467-9.

21. Kiellberg Larsen G, Haedersdal M, Svejgaard EL. The prevalence of onychomycosis in patients with psoriasis and other skin diseases. Acta Derm Venereol. 2003; 83: 206-9.

22. Saunte DML, Holgersen JB, Haedersdal M, Strauss G, Bitsch M, Svendsen OL et al. Prevalence of toe nail onychomycosis in diabetic patients. Acta Derm Venereol. 2006; 86: 425-8.

23. Szapietowski JC, Reich A, Garlowska E, Kulig M, Baran E. Factors influencing coexistence of toenail onychomycosis with tinea pedis and other dermatomycoses. Arch Dermatol. 2006; 142: 1279-84. 